\title{
A Probabilistic Fatigue Model for Quasi-Brittle Materials
}

\author{
YU Rena C., SAUCEDO Luis and RUIZ Gonzalo \\ University of Castilla-La Mancha, Ciudad Real, Spain \\ rena@uclm.es
}

\begin{abstract}
We aim to develop a new fatigue model valid for quasi-brittle materials like concrete, which properties have considerably larger standard deviation than metals. Having this in mind, we fit the measured strength data with a three-parameter Weibull cumulative distribution function and in turn take it as the initial distribution for an asymptotic fatigue model in concrete. We also take into account the observed influence of frequency and stress ratio on the fatigue life in concrete, both plain and reinforced with fibers. The developed model is validated against fatigue tests in compression on cubic specimens for different stress ratios and loading frequencies. The secondary strain rate is also found to be correlational with the number of cycles to failure.
\end{abstract}

Index Terms - Fatigue, frequency effect, probabilistic model, secondary strain rate

\section{Introduction}

Interest in the fatigue of concrete began more than a hundred years ago with the development of reinforced concrete bridges. Since then, numerous experiments have been conducted to study the influence of different fatigue parameters. For metals, the stress level plays an important role, and the fatigue life (the number of cycles $\mathrm{N}$ resisted before failure) is often described by the S-N curves. For concrete, however, the influence of the stress ratio, loading frequency and stress level has been observed to be important.

In the current work, we first consider the entire statistical distribution given by the characterization tests and build our fatigue model from this initial distribution. Second we take into account the loading frequency based on the dynamicresponse description given by the Model Code 2008 [1]. The range of application of the proposed model is below $10 \mathrm{~Hz}$ according to the experimental tests realized between 1/16 and $4 \mathrm{~Hz}$ of Ruiz et al [2]. Even though the model itself does not limit to a given range of frequency, its application beyond 10 $\mathrm{Hz}$ needs further experimental collaboration.

The rest of the paper is organized as follows. A fatigue model based on an initial distribution is postulated next. Then the experimental program and validation of the model are given before relevant conclusions are drawn.

\section{The Fatigue Model with an Initial Distribution}

As mentioned before, we aim to develop a fatigue model for concrete, taking into account the statistical distribution of the characterized strength data and the influence of loading frequency and stress ratio, the following hypotheses are assumed.

a) The characterized (or experimentally measured) material property of concrete, such as the compressive or tensile strength, follows a Weibull distribution. In the current work, we focus on the compressive strength measured from cubic specimens.

b) This distribution is influenced by the dynamic condition through the loading frequency. In addition, the relation given by the Model Code to describe the dynamic properties of concrete is extendable to consider the influence of loading frequency according to the experimental data of Ruiz et al. [2].

c) There exists a minimum stress which is the asymptote given by the zero probability of failure.

Given sufficient number of characterization tests carried out at a certain reference loading rate, the probability of failure (PF) corresponding to each stress level can be fitted by a threeparameter Weibull CDF as follows

$$
P F\left(\sigma_{f_{0}}\right)=1-\exp \left[-\left(\frac{\sigma_{f_{11}}-\sigma_{\text {min }}}{\lambda}\right)^{k}\right], \sigma_{f_{0}} \geq \sigma_{\min }
$$

The meaning of each parameter is given in the nomenclature section. The relation between the static and dynamic compressive strength given by the Model code is extended to include the loading frequency effect through the following equation

$$
\frac{f_{c d}}{f_{c o}}=\left(\frac{\dot{\sigma}_{d}}{\dot{\sigma}_{a}}\right)^{\alpha} \quad \alpha=0.014 \exp [\gamma f] \quad \dot{\sigma}_{\dot{d}}=2 f \Delta \sigma
$$

By fitting the curve iso-probability of failure through the following equation

$$
\begin{aligned}
& \sigma_{f}=\sigma_{\text {mina }}+\left(\sigma_{f_{0}}-\sigma_{\text {mino }}\right) N^{-\sigma(1-f i)} \\
& a=b+c \ln (1+f)
\end{aligned}
$$

we can establish the input-output distribution shown in Fig. 1. 


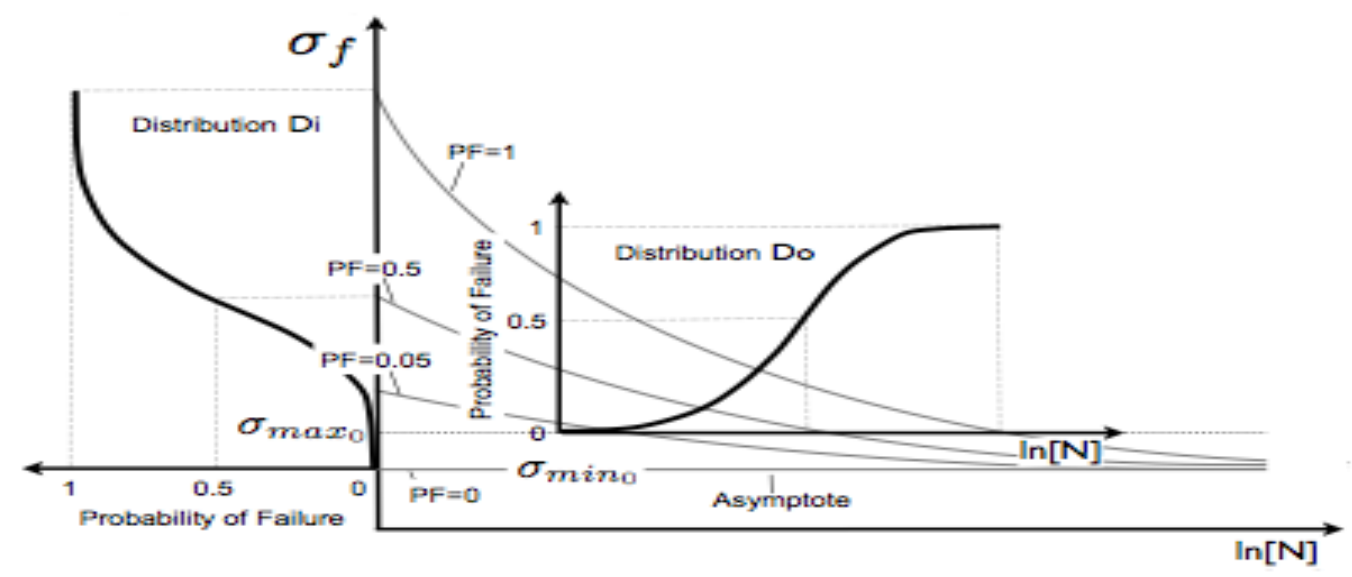

Figure 1: Failure curves of iso-probability, where $D$, is the initial CDF, determined from characterization tests and fited acoording to Eq. 2, whereas $D_{0}$ is the output CDF fitted by the fatigues tests.

\section{Experimental Program and Model Validation}

The experimental program was designed to look into the influence of the stress ratio and loading frequency on the fatigue strength of concrete. Two groups of tests on two different types of concrete $\mathrm{C} 1$ and $\mathrm{C} 2$ were carried out for cubic specimens. Twenty compressive tests on cubes $(80 \mathrm{~mm}$ in edge length) for $\mathrm{C} 1$, and six compressive tests for cubes

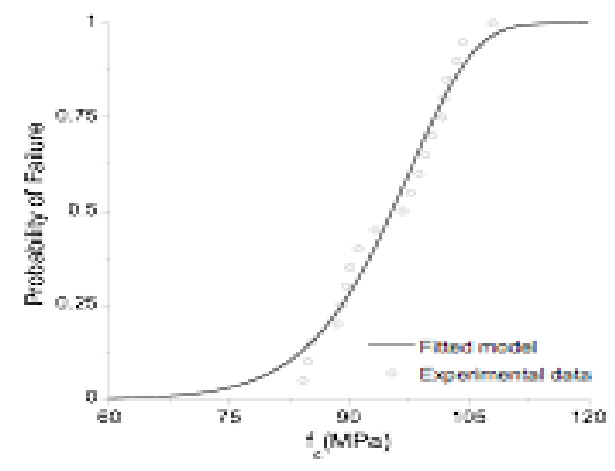

(100 mm in edge length) for $\mathrm{C} 2$, were carried out for characterization purpose. In addition, two groups of tests also were performed on fiber reinforced concrete $\mathrm{CF} 1$ and $\mathrm{CF} 2$. The measured strength variation for $\mathrm{C} 1$ and $\mathrm{C} 2$ is fitted through the Weibull distribution as shown in Fig. 2. The relation between secondary strain rates and fatigue life for $\mathrm{CF} 1$ and CF2 is demonstrated in Fig. 3.

Figure 2: Initial distribution of the compressive strength fitted with the experimental measurements on cubes for concrete $\mathrm{C} 1$ (left) and $\mathrm{C} 2$ (right).
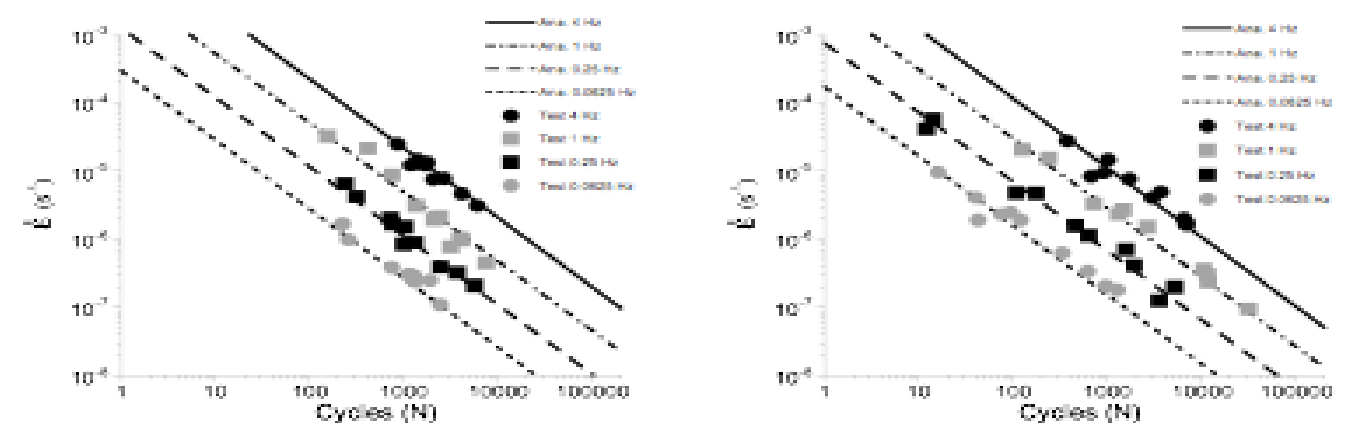

Figure 3: Secondary strain rate plotted against the number of cycles resisted for CF1 (left), CF2 (right) at four loading frequencies. 


\section{Nomenclature}

- $\sigma_{\operatorname{maz}}$ and $\sigma_{\min }:$ Maximum and minimum stress.

- $\triangle \sigma$ : Stress range $\sigma_{m a a^{-}}-\sigma_{\text {rnir- }}$

- $R$ : Stress ratio defined as $\sigma_{\min } / \sigma_{\max }$.

- $\sigma_{\operatorname{man} 0}:$ Static equivalence of $\sigma_{\max }$.

- $\sigma_{f}$ : Failure stress.

- $\sigma_{f_{0}}$ : Intercept of the IPFC with the $\sigma_{f}$-axis.

- $f_{C_{\mathrm{d}}}$ and $f_{\mathrm{C}_{\mathrm{D}}}$ : Dynamic and static compressive failure stress

- $\sigma_{\min }:$ Horizontal asymptote which determines the lower stress value.

- $\dot{\sigma}_{d}$ : Loading rate of the fatigue test.

- $\sigma_{c}$ : Critical stress.

- $N$ : Number of cycles to failure.

- $\lambda, k:$ : Scale and shape parameter of the Weibull distribution.

- PF: Probability of failure in any point of the domain $\sigma_{f}-\ln N$.

- $f$ : Loading frequency of a fatigue test.

- $f_{0}$ : Reference loading frequency.

- $a, b$ and $c$ : Parameter that adjusts the relation between $\ln N$ and $f, R$.

- $\dot{\varepsilon}$ : Secondary strain rate in a fatigue test.

- $\alpha$ : Exponent that measures the amplification of dynamic strength.

- $\gamma$ : Coefficient that takes into consideration of loading frequency for $\alpha$.

\section{Conclusions}

By taking into consideration the dynamic properties of concrete, fitting the results of material characterization tests with a Weibull distribution and assuming it as the initial distribution, which can be shifted along the failure axis, we have developed a fatigue model which is capable of dealing with different frequencies and stress ratios for two plain concretes and two concretes reinforced with fibers. The model is validated against a total of 153 fatigue tests for two plain high strength concrete and two concretes reinforced with steel or polypropylene fibers, performed at two different stress ratios and four different loading frequencies. In addition, we have shown the failure is probabilistic in terms of the number of cycles $\mathrm{N}$ or the secondary strain rate, but there is a one-toone relation between $\mathrm{N}$ and secondary strain rate. In practical terms, this provides the possibility of determining the fatigue life $\mathrm{N}$ without actually exhausting all the cycles.

\section{References}

[1] CEB-FIP. Constitutive modeling of high strength/high performance concrete. FIB Bulletin 42, (2008).

[2] G. Ruiz, A. Medeiros and X.X. Zhang. Experimental study of loading frequency effect on compressive fatigue behavior of plain and fiber reinforced concretes. Anal. Meca. Fract., Vol. 29 (2011), p. 535-540. 